\title{
Experiences in set-up and usage of a geodetic real-time differential correction network
}

\author{
Sven Martin ${ }^{1}$ and Cord-Hinrich Jahn ${ }^{2}$ \\ ${ }^{1}$ Institute of Flight Guidance and Control, Technical University of Braunschweig, Germany \\ ${ }^{2}$ State Survey Office of Lower Saxony, Hannover, Germany
}

(Received December 1, 1999; Revised June 6, 2000; Accepted June 6, 2000)

\begin{abstract}
Global Navigation Satellite Systems (GNSS) are commonly used for geodetic and land surveying applications. The stand alone accuracy provided by these GNSS are insufficient for the majority of these operations (GPS, 1995), therefore some form of differential correction method is required. Accordingly, the state survey offices of Germany have installed a differential correction service for geodetic applications. Code- and phase-corrections are broadcast in the VHF-band using the RTCM V2.1 format (RTCM, 1994). One major problem is that the accuracy depends on the distance to a reference station (length of baseline) because of residual orbit and atmospheric biases. To achieve a more precise solution, a number of reference stations are connected together to form a network. Within this network these influences are computed and a set of "area correction parameters" are also transmitted in RTCM message Type 59. Field trials and measurements have confirmed the high accuracy of this service. This paper describes the system itself, investigations of communication methods as well as site planning. In addition measurements from field trials will be presented to demonstrate the high accuracy in a real-time environment.
\end{abstract}

\section{Introduction}

Global Navigation Satellite Systems (GNSS) have become more and more important for geodesy and land surveying. Unfortunately, on their own, they are not accurate enough for precise operations. To gain more accurate position solutions a popular method that is used in surveying is known as "Real Time Kinematic (RTK)". RTK allows 'on-the-fly' ambiguity fixing by using correction data from a reference receiver (which has to be installed close to the surveyed area). The GPS receiver has to be placed on a coordinated reference point and needs a sufficiently powerful radio transmitter to broadcast across the whole survey area.

Such installations are costly and take a lot of time to establish. To obtain a more cost-effective solution a modified concept for a geodetic differential service has been developed. The first step was to install several reference stations on precisely located points, in the European Terrestrial Reference Frame (ETRF) datum. Each station is equipped with a geodetic quality, dual-frequency GPS receiver, a computer running reference station software to calculate differential corrections, and a radio transmitter to broadcast these differential corrections. The mobile user needs a differentiallycapable GPS unit and a VHF receiver. In 1991 a project was launched to set-up a near-online geodetic DGPS service in the federal state of Lower Saxony (HPPS) (Jahn, 1997). Since 1995 a real-time service has been operating nationwide that transmits data with an update rate of $1 \mathrm{~Hz}$ using the RTCM V2.1 format. The user is able to choose between two message categories: a) Code correction, accuracy approximately

Copy right (C) The Society of Geomagnetism and Earth, Planetary and Space Sciences (SGEPSS); The Seismological Society of Japan; The Volcanological Society of Japan; The Geodetic Society of Japan; The Japanese Society for Planetary Sciences.
1-5 m (Message Type 1); and b) Phase correction, accuracy approximately 1-5 cm (Message Type 20/21). Positioning accuracy is dependent upon the GPS receiver quality.

The accuracy of these kinds of corrections is limited to 1 to $10 \mathrm{ppm}$ of the baseline length due to satellite orbit errors and atmospheric effects (Wanninger, 1995). The ability to resolve the ambiguities 'on-the-fly' is limited to a baseline length of a few kilometres. In order to increase the working range and to reach a more precise and faster solution, several reference stations are connected together to form a real-time multi-station network. Carrier-phase data from each GPS reference receiver is processed with specially developed software, which calculates a set of so-called "Area Correction Parameters (ACP)" (Wuebbena et al., 1996a). These area correction parameters model the distance dependent errors, and are also broadcast in RTCM Message Type 59. The inter-receiver data connection is by directional microwave links, which means that there is no need for additional and expensive leased lines.

\section{Concept of a High Precision Real-Time Differ- ential Correction Service}

The basic idea of a permanent High Precision Positioning Network is to install several GPS receivers on precisely surveyed reference points, connect them together by radio links (or leased lines), and to calculate area correction parameters (Fig. 1). These parameters are transmitted to the user, along with differential code and phase corrections. Each reference station is monitored and controlled by a central master reference station, where data is collected and stored. All GPS reference stations are permanently up and broadcasting. Much research has been carried out to investigate the optimum distance between the reference stations, because 


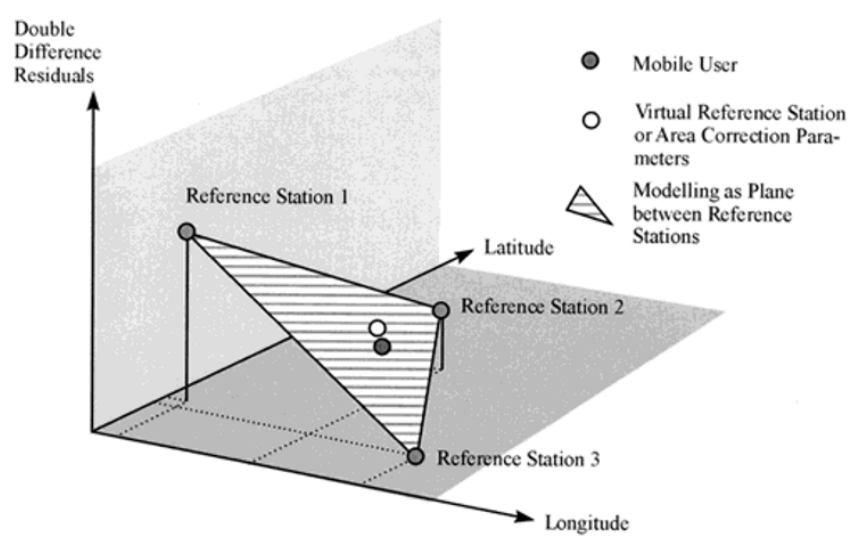

Fig. 1. Modelling of the area correction parameters.

the systematic errors increase with the length of baseline. So an optimum spacing needs to be found to balance between a maximum coverage area, minimum number of reference stations and the minimum distance to the user within this network. Froehlich (1995) and Jahn (1997) suggest distances from reference station to reference station of approximately 40-60 km when using 6 visible satellites and observation times of 10-15 minutes.

\subsection{The reference station}

Every station is equipped with a high quality, geodetic 12-channel L1/L2 GPS receiver and a choke-ring antenna on a precisely determined reference point $(\ll 1 \mathrm{~cm})$. These reference points are included in, or derived from, the European Terrestrial Reference Frame (ETRF). The elevation limit should be at least $5^{\circ}$. Siting criteria are: free horizon, no reflectors, no radars and especially no amateur radio links (which may cause significant loss of signal/noise on the L2-carrier).

GPS raw data is fed into a computer running the reference station software. At present there are two different software packages being used (GNREF $\left(\mathrm{GEO}^{++}, 1997\right)$ and GPSbase (Spectra, 1998)). Each generates RTCM V2.1 differential corrections. Message Types 1, 3, 14, 16, 20, 21 are generated. Due to the small bandwidth and low data rate on the radio transmission link, RTCM Message Type 20 and 21 are compressed and broadcast using RTCM Message Type 59. Decompression and conversion from Type 59 to Type 20/21 is done by a hardware decoder at the mobile user which has to be inserted into the data stream from the DGPS radio receiver. Many GPS receivers already support the correction data interface RTCM V2.1 with the Message Types 20 and 21. Data is additionally recorded (interval $1 \mathrm{sec}$ ) and stored (15 sec) in RINEX format and can be retrieved via mobile telephone (GSM) in near-real-time or for post-processing applications. Every reference station broadcasts its differential corrections via a VHF radio modem with an update rate of 1 Hz. Figure 2 shows the equipment and communication links.

After specifying the basic concept of the differential correction service, the installation of reference stations began. First of all, suitable locations had to be investigated. Radio and network planning was done with respect to line of sight coverage, receiver/transmitter height and earth curva-

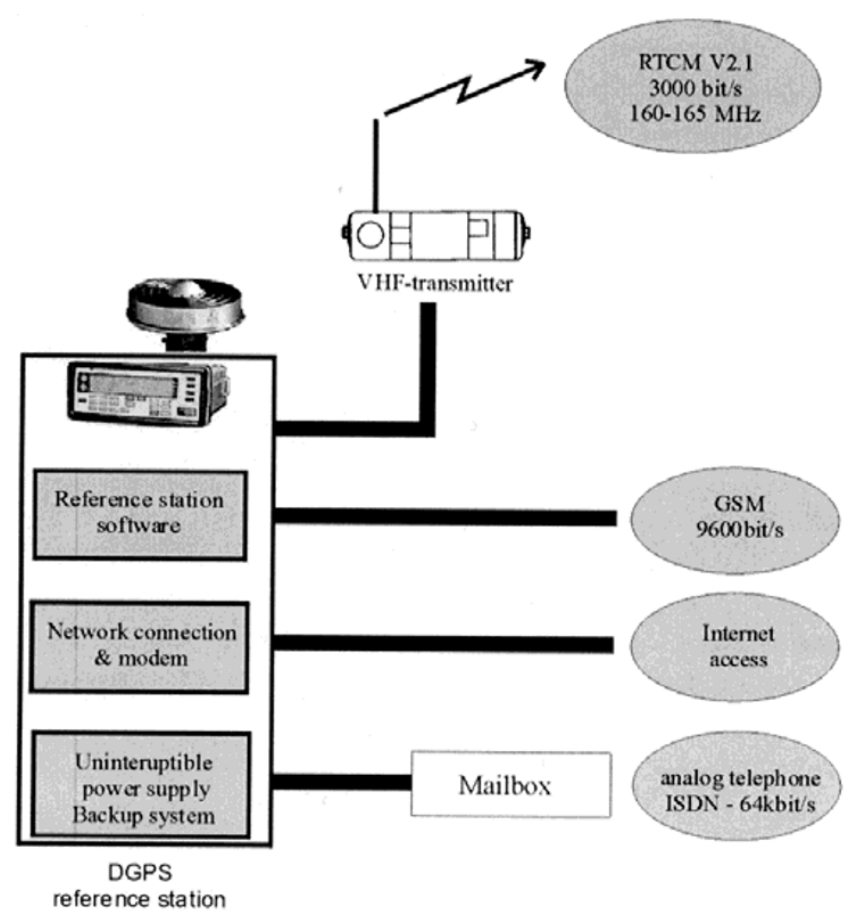

Fig. 2. Reference station equipment.

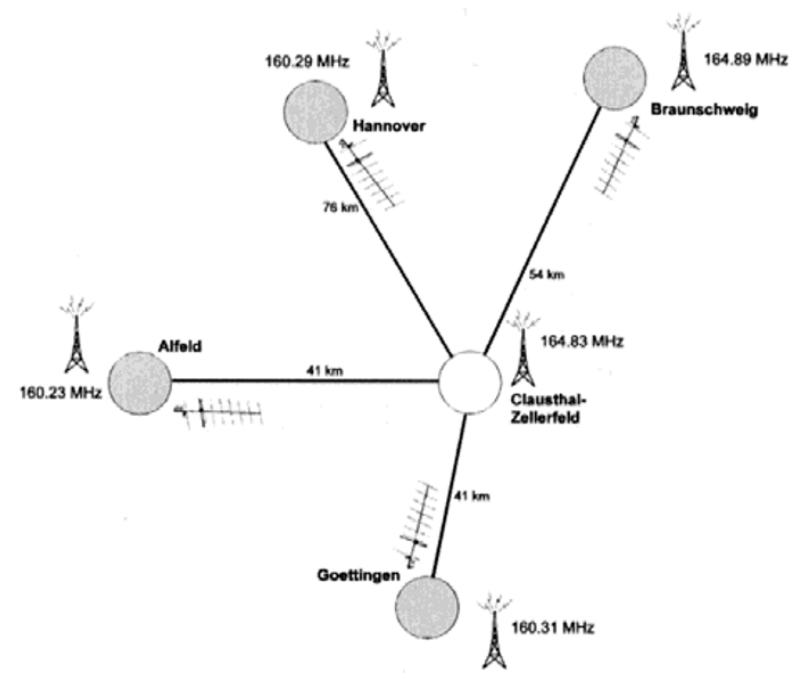

Fig. 3. System concept of the high precision real-time differential correction network.

ture. The network concept plays an important role in radio planning, because every reference station needs a directional microwave link to the master station (see Fig. 3). Carrierphase data from each reference station is transmitted via a directional radio link to the master station. The master station computes the area correction parameters and broadcasts them in RTCM Message Type 59. Each reference station extracts these parameters from the received data stream and applies them to its own differential corrections. A more detailed description of the system can be found in Jahn (1997), Froehlich (1995) and Augath (1998). 


\section{Technical Specifications and Data Communica- tion Link}

The technical specification of a data broadcast service deals with coverage, frequency allocation, bandwidth and transmitter power. VHF and UHF band can be used. The advantage of VHF is the wider coverage in open area, whereas UHF is better for urban areas because of the better penetration through buildings. Communication link issues are discussed in the following sections.

\subsection{Private mobile radio}

Private mobile radio (PMR) was first introduced as a communication method for companies in local areas, e.g. taxiservices, transportation, building contractors, private security services, etc. No user infrastructure is needed for communication between two radios. There are two different frequency bands reserved for PMR, one in the VHF-band from $140 \mathrm{MHz}$ to $170 \mathrm{MHz}$ and the other in the UHF-band between 430-470 MHz. Channel spacing is $12.5,20$ and 25 $\mathrm{kHz}$. Due to the local character of PMR, frequencies are reallocated every few kilometres. In the last few years the usage of PMR has rapidly increased so that frequencies have already been allocated to different users. PMR does not provide any privacy (encryption is not implemented). Small bandwidth and slow data communication rates make it difficult to use PMR for a DGPS service. Using Fast Frequency Shift Keying (FFSK) modulation a data rate of up to 3000 bit/s can be achieved. Newer radio modems use Gaussian Minimum Shift Keying (GMSK), and communication rates of $19.2 \mathrm{kbit} / \mathrm{s}$ are possible. Advantages are low cost, easy installation and operation.

\subsection{Digital Audio Broadcast (DAB)}

Digital Audio Broadcast (DAB) is an international broadcasting standard developed under the EUREKA-147 Project. DAB is a multi-service broadcasting system that can be used on terrestrial, satellite, hybrid (satellite with complementary terrestrial transmission) and cable broadcasting networks. The system is designed for mobile, portable and fixed receivers. It features a flexible digital multiplex that can carry a number of services, both audio and independent data. In December 1994 DAB was designated a worldwide standard in ITU Recommendations BS 1114 and BO 1130, for terrestrial and satellite sound broadcasting respectively, to vehicular, mobile and fixed receivers in the VHF/UHF frequency range. In Germany many test facilities have been installed. $\mathrm{DAB}$ is the most promising wide-area broadcast method for the future. Some state surveying offices have already set up DGPS broadcast by DAB, but tests are still in progress. Problems have occurred with the continuity of the data stream and the age of data (from the GPS reference station to mobile users). Data is inserted in the DAB data stream by a statistical multiplexer. However, this procedure cannot guarantee a constant delay because the load of the audio stream varies, and data services are only used as 'gap fillers'.

\subsection{Mobile phones (GSM)}

As shown in Fig. 2 each station is accessible by mobile phone. In the beginning this was only for near-real-time or post-processing applications to retrieve data while performing field measurements.

The reference station software has since been modified to broadcast real-time differential correction data via mobile phones. There is a login procedure required to gain access to the data. A communication software handles incoming calls and provides the correction data to the user. The disadvantage is the limited number of parallel users on a reference station.

From the technical point of view, in Germany there are two different types of digital cellular phone networks: GSM 900 (D1 and D2) and GSM 1800 (E-Netz) (GSM-Global System for Mobile Communication). Analogue cell phone services ceased operation in 1996. Data communication is possible in both GSM networks with a maximum data rate of $9600 \mathrm{bit} / \mathrm{s}$. To minimize cost the reference station is equipped with a mobile phone as well. Hence dialing in from a mobile phone while on a field trial is an inter-network connection, which is much cheaper than calling a fixed line phone from a mobile phone. The cell phones must be prepared for data communication (in fact most of GSM 900 and GSM 1800 devices are capable of data transfer). A comparative study between VHF radio broadcast and GSM has shown that up to a usage time of approximately $5000 \mathrm{~min} / \mathrm{year}$ the cell phone access is more economic than VHF broadcast, due to the very low purchase price of cell phones. (The price of phones are subsidized by the service providers.) Further advantages are that there is no need to apply for radio frequencies, and the reduced installation cost. In future the user could be identified by his cell phone number, which is transmitted on every call (caller line identification). The login procedure then becomes obsolete.

Another technological advance is UMTS (Universal Mobile Telecommunication System). Development for this future standard of mobile communication is in progress, and data transfer should be possible at rates of up to $2 \mathrm{Mbit} / \mathrm{s}$. A higher data rate becomes important with respect to future possible integration of GLONASS and GALILEO.

\subsection{Installed communication link}

Initially, installations were made with Private Mobile VHF radio modems with a data rate capability of approximately $3000 \mathrm{bit} / \mathrm{s}$. The choice of PMR was due to the advantages

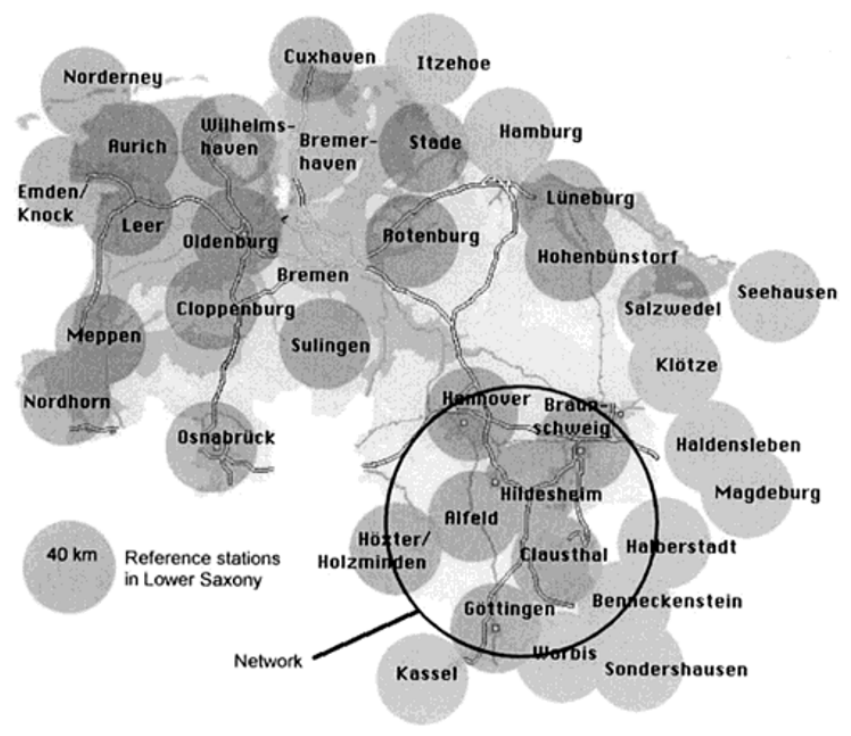

Fig. 4. Reference stations in Lower Saxony and neighbouring states (LGN, 1999). 


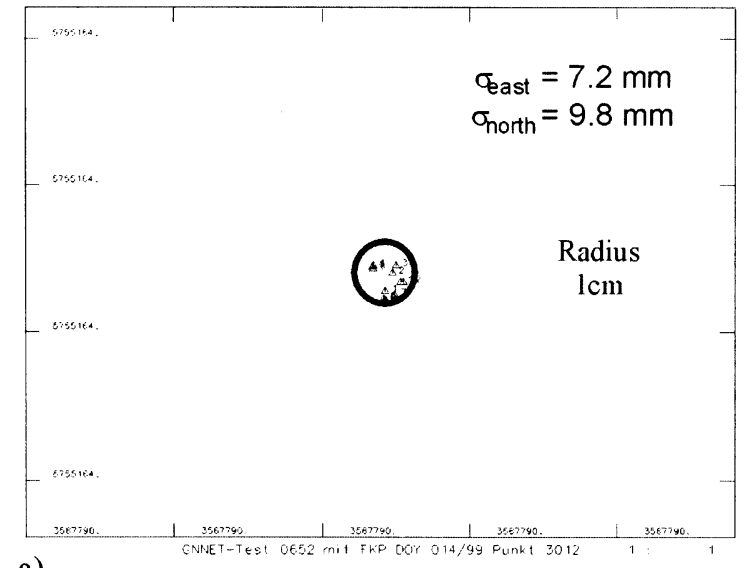

a)

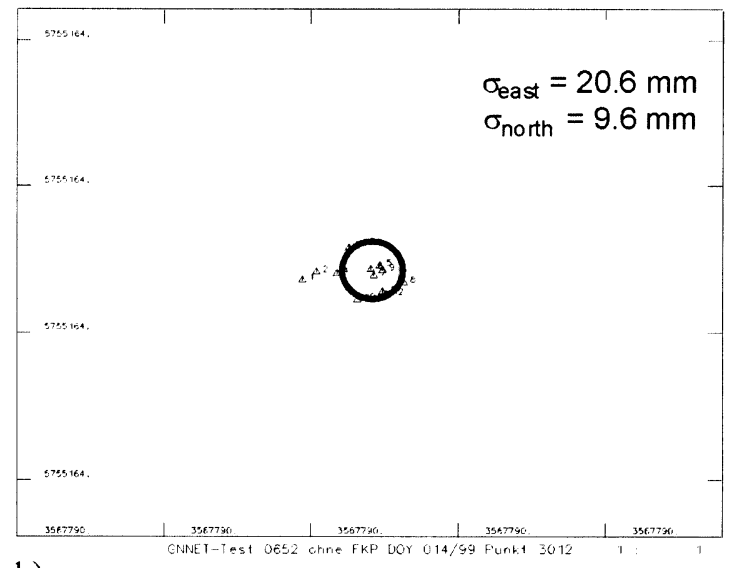

b)

Fig. 5. a) Measurement with ACP, distance to nearby reference station $15 \mathrm{~km}$. b) Measurement without ACP (RTCM Type $20 / 21$ only).

of low cost, easy installation and low maintenance (as mentioned in Subsection 3.1). Communication specifications of the real-time differential correction network are as follows:

- RF-data rate: $3000 \mathrm{bit} / \mathrm{s}$

- Modulation technique

FFSK

- Frequency 160.23-164.89 MHz

- No. of channels 5

- Transmitter power $25 \mathrm{~W}$ Figure 4 shows the currently operating reference stations in Lower Saxony. The circled stations in the south are connected together and form the network described here.

\section{Test Measurements and Results}

The first operational, real-time differential correction network in Germany is located in the south of Lower Saxony. There are 5 reference stations: Hannover, Braunschweig, Goettingen, Alfeld and Clausthal-Zellerfeld (see Figs. 3 and 4). Clausthal-Zellerfeld is the master station where the area correction parameters are calculated. Test measurements were carried out in 1999 within this geographic polygon. A Trimble SSi GPS receiver was used together with a Trimble Geodetic antenna (Compact L1/L2, TRM14532.00, with ground plane).

A number of points with different distances to the reference stations were used for testing the new algorithms. Distances between 5 and $40 \mathrm{~km}$ with a grid of $5 \mathrm{~km}$ were considered. Figure 5 shows the coordinate variations of a point in the centre of the network. Each point represents a position fix. After fixing the ambiguities the search has been restarted. The distance to the nearest reference station was approximately $15 \mathrm{~km}$, but with Area Correction Parameters (ACP) this is not really a baseline (without ACP, it is a true baseline). The diagram shows an accuracy of the order of 1-2 cm with ACP (Fig. 5(a)), becoming a little worse without ACP (Fig. 5(b)).

In general, the accuracy varies in the horizontal component by about a few millimetres for points close to the reference station, to a few centimetres for longer distances (Jahn, 1999).

The results of the field measurements have shown the ad-

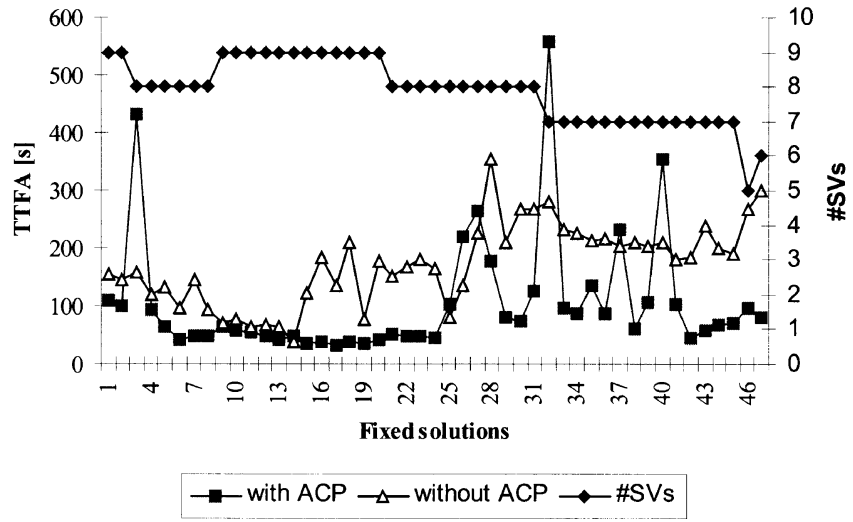

Fig. 6. Time-To-Fix-Ambiguities (TTFA).

vantage of the real-time algorithm. Positioning over 30-40 $\mathrm{km}$ is possible within 1-2 minutes with accuracies of about $\pm 1 \mathrm{~cm}$. Comparing the TTFA (Time To Fix Ambiguities) to normal RTK solutions the time is decreased significantly (Fig. 6). Multipath at the mobile user and undetermined phase centre variations of the GPS antenna still deteriorate the results (Menge, 1998; Jahn, 1999). Figure 7 shows results for a point with a distance of $40 \mathrm{~km}$ to the nearest reference station. With ACP the accuracy is about $4-5 \mathrm{~cm}$. However, ambiguity fixing without ACP was not possible even with observation times of about $15 \mathrm{~min}$.

\section{Conclusions and Outlook}

This paper has demonstrated the high accuracy of a multistation DGPS reference network. Distance dependent errors have been reduced to a minimum and the Time To Fix Ambiguities (TTFA) has decreased significantly.

Due to this technique, cost and surveying time can be reduced considerably because the user needs only one mobile GPS receiver and a VHF radio modem. Future investigations will deal with more efficient communication links, higher data rate and bandwidth, and improved modulation techniques. With respect to an optional integration of GLONASS or GALILEO, it is necessary to test new radio link technolo- 


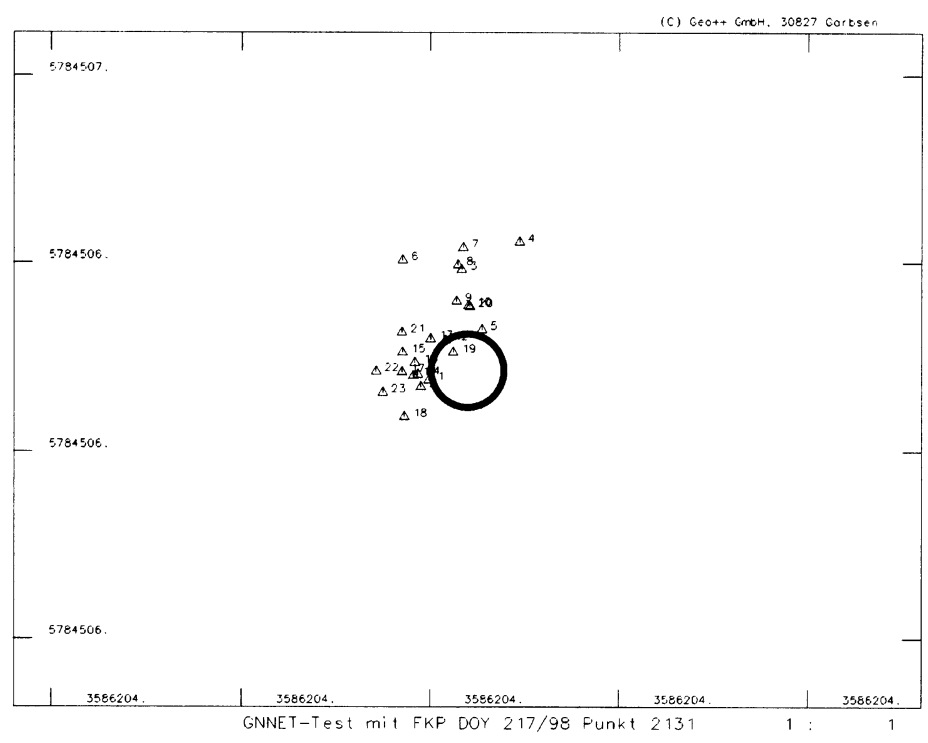

Fig. 7. Measurement with ACP. Distance to nearby reference station $40 \mathrm{~km}$. Variations due to local multipath and high ionospheric activity (data was collected during noon).

gies because more satellites require greater bandwidth. DAB and UMTS will likely play an important role in future.

Furthermore, in view of increasing solar activity, additional investigations on the error modelling of the ionosphere are required. Wanninger (1999) has shown that the performance of ambiguity resolution can be enhanced by regional modelling of the ionospheric error. Another field of interest will be antenna phase centre variations and calibration of these offsets (Wuebbena, 1996b; Menge, 1998; Geiger, 1998). The installation of reference stations will continue and within the next months further networks will be linked together.

Acknowledgments. The project "High-Precision Permanent Positioning Service (HPPS)" is supported by the German Aerospace Center (DLR) under grant No. 50 ND 9602.

\section{References}

Augath, W. and C.-H. Jahn, The High-Precision Positioning Service (HPPS) and its Application in Cadastral Surveying in Germany, Proceedings of 21. FIG Meeting in Brighton, Commission 5, pp. 9-19, 1998.

Froehlich, M., Zur Entwicklung eines Hochpraezisen Permanenten Positionierungsservice (HPPS), Wiss. Arb. d. Fachr. Vermessungswesen der UNI Hannover Nr. 211, 1995.

Geiger, A., Modeling of phase center variation and its influence on GPSPositioning, Intern. GPS-Workshop Darmstadt, April 1998.

$\mathrm{GEO}^{++}$, Product information of the software module GNNET and GNREF, 1997.
GPS, Global Positioning System: Standard Positioning Service Signal Specification, 2. Rev., July 1995

Jahn, C.-H., Der hochpraezise permanente Positionierungs-Service (HPPS), Ortung und Navigation 1/1997, pp. 48-73, 1997.

Jahn, C.-H. and U. Feldmann-Westendorf, SAPOS für Liegenschaftsvermessungen, Proceedings of the 2nd SAPOS Symposium, Berlin, 1999.

LGN, Landesvermessung und Geobasisinformation Niedersachsen, http: / / www. Ign. de, 1999.

Menge, F., G. Seeber, C. Voelksen, G. Wuebbena, and M. Schmitz, Results of Absolute Field Calibration of GPS Antenna PCV, Proceedings of the ION GPS 98, Nashville, U.S.A., pp. 31-38, 1998.

RTCM Recommended Standards for Differential GNSS Service, Version 2.1, RTCM Special Committee No. 104, Alexandria, 1994.

Spectra, Spectra Precision Terrasat GmbH: Product Information, 1998.

Wanninger, L., Improved Ambiguity Resolution by Regional Differential Modelling of the Ionosphere, Proceedings of the ION GPS 95, Palm Springs, pp. 55-62, 1995.

Wanninger, L., The Performance of Virtual Reference Stations in Active Geodetic GPS-networks under Solar Maximum Conditions, Proceedings of the ION GPS 99, Nashville, pp. 1419-1427, 1999.

Wuebbena, G., A. Bagge, G. Seeber, V. Boeder, and P. Hankemeier, Reducing Distance Dependent Errors for Real-Time Precise DGPS Applications by Establishing Reference Station Networks, Proceedings of the ION GPS 96, Kansas City, pp. 1845-1852, 1996a.

Wuebbena, G., F. Menge, M. Schmitz, G. Seeber, and C. Voelksen, A New Approach for field calibration of absolute antenna phase center variations, Proceedings of the ION GPS 96, Kansas City, pp. 1205-1214, 1996b.

S. Martin (e-mail: s.martin@tu-bs.de) and C.-H. Jahn (e-mail: cordhinrich.jahn@lgn.niedersachsen.de) 